\title{
Consensus of a kind of Dynamical Agents in Network with Time Delays
}

\author{
Hongwang Yu, Baoshan Zhang \\ School of Mathematics and Statistic, Nanjing Audit University, Nanjing, China \\ E-mail: yuhongwang@nau.edu.cn \\ Received August 4, 2010; revised September 18, 2010; accepted October 23, 2010
}

\begin{abstract}
This paper investigates the collective behavior of a class of dynamic agents with time delay in transmission networks. It is assumed that the agents are Lyapunov stable distributed on a plane and their location coordinates are measured by some remote sensors with certain error and transmitted to their neighbors. The control protocol is designed on the transmitted information by a linear decentralized law. The coordination of dynamical agents is shown under the condition that the error is small enough. Numerical simulations demonstrate that our theoretical results are valid.
\end{abstract}

Keywords: Multi-Agent System, Time Delay, Consensus Protocol

\section{Introduction}

Distributed coordination of network of dynamic agents has attracted a great attention in recent years. Modeling and exploring these coordinated dynamic agents have become an important issue in physics, biophysics, systems biology, applied mathematics, mechanics, computer science and control theory [1-10]. How and when coordinated dynamic agents achieve aggregation is one of the interesting topics in the research area. Such problem may also be described as a consensus control problem.

To describe the collective behavior of agents in a large scale network, the agent in the network usually is modeled by a very simple mathematical model, which is an approximation of real objects. Saber and Murray [3,4] proposed a systematical framework of consensus problems in networks of dynamic agents. In their work the dynamics of the agent is modelled by a simple scalar continuous-time integrator $\dot{x}=u$, the convergence analysis is provided in different types of the network topologies. Following the work of [2,3], G. Xie and L. Wang [8] study the case where the dynamics of each agent is second order. In their work, they show that by means of a simple linear control protocol based on the structure of the graph, the dynamical agents will eventually achieve aggregation, i.e., all agents will gradually move into a fixed position of the line, meanwhile their velocities converge to zero.
In networks of the dynamic agents, time delays and sensor error may arise naturally, e.g., because of the moving of the agents, the congestion of the communication channels and the finite transmission speed due to the physical characteristics of the medium transmitting the information. The different protocols with time delays have been investigated [2,4]. And the sensor error or communicated errors are also to be considered in the consensus protocol [9]. It is shown that the collective behavior of dynamical agent will depend on the communicated error and the algebraic characterization of the communicated network topology.

In this paper, we study the consensus of multiagent systems where the dynamics of each agent is second order. The agents may represent the vehicles or mobile robots spread over a wild area and they communicate by means of some remote sensors with certain error and time delay. When the agents are moving in a plane, the consensus conditions will depend on the time delay, communicated error and the algebraic characterization of network topology, as well as the dynamical behavior of agents.

This paper is organized as follows. In Section 2, we recall some properties on graph theory and give the problem formulation. In Section 3 the main results of this paper are given and some simulation results are presented in Section 4. Final section is a conclusion. 


\section{Preliminaries}

By $G=(V, E, A)$, we denote an undirected graph with an weighted adjacency matrix $A=\left[a_{i j}\right]$, where $V=\left\{p_{1}, \cdots, p_{M}\right\}$ is the set of nodes, $E \subseteq V \times V$ is the set of edges. The node indexes belong to a finite index set $\underline{M} \triangleq\{1, \cdots, M\}$. An edge of $G$ is denoted by $e_{i j}=\left(p_{j}, p_{i}\right)$. The adjacency elements $a_{i j}$ are defined in following way: $e_{i j} \in E$ $\Leftrightarrow a_{i j}>0$. Moreover, we assume $a_{i i}=0$ for all $i \in \underline{M}$. The set of neighbors of node $p_{i}$ is denoted by $N_{i}=\left\{p_{j} \in V \mid\left(p_{i}, p_{j}\right) \in E\right\}$.

A diagonal matrix $D=\operatorname{diag}\left\{d_{1}, \cdots, d_{M}\right\}$ is a degree matrix of $G$ with $d_{i}=\sum_{j=1}^{M} a_{i j}$. Then the Laplacian of the weighted graph $G$ is defined as $L=D-A$. A graph is called connected if there exists a path between any two distinct vertices of the graph.

An important fact of $\mathrm{L}$ is that all the row sums are zero, therefore, $1_{M}=(1, \cdots, 1)^{T}$ is an eigenvector of $L$ associated with zero eigenvalue. Moreover, the graph $G$ is connected if and only if its Laplacian $L$ is satisfied $\operatorname{rank}(L)=M-1$ and all eigenvalues of $L$ are of positive real numbers except that only one eigenvalue is zero [2].

Consider a network of dynamical agents defined by a graph $G=(V, E, A)$. The node set $V$ consists of dynamical agents $P_{i}, i \in \underline{M}$. Let $x_{i}=\left(x_{i 1}, x_{i 2}\right)^{T} \in R^{2}$ be the coordinate of dynamical agent $P_{i}$, then the dynamics of $p_{i}$ are identical and described as follows.

$$
\begin{aligned}
\dot{x}_{i} & =v_{i} \\
m_{i} \dot{v}_{i} & =K v_{i}+u_{i} \\
y_{i} & =F\left(\begin{array}{l}
x_{i} \\
v_{i}
\end{array}\right)
\end{aligned}
$$

where $x_{i}$ is indicates the location vector of agent $p_{i}$ in the plane, $v_{i}=\left(v_{i 1}, v_{i 2}\right)^{T}$ represents its velocity vector of the ith agent, $m_{i}$ is its mass and $K=\left[\begin{array}{ll}k_{11} & k_{12} \\ k_{21} & k_{22}\end{array}\right]$ is a dynamical feedback matrix of the agent. $F$ is an observation matrix of the agent by some remote sensor. In what follows we simply assume that $m_{i}=1$ and $p_{i}=x_{i}$. Let $F=\left[\begin{array}{ll}C & 0\end{array}\right]$ which means that the location information of the ith agent is only measured by some remote sensor and is transmitted to its neighbors through the network. The matrix $C$ is assumed to be the form $C=\left[\begin{array}{cc}1 & \sigma_{i} \\ -\sigma_{i} & 1\end{array}\right]$. The parameter $\sigma_{i}$ indicates that the network communicated error or the coordinates used for sensor could be different from that of the agents.

For the dynamic agent (1) in network we have following assumption.
Assumption 1. The dynamics (1) is Lyapunov stable when it disconnected with its neighbors, meaning that the dynamical agent as an autonomous will gradually stop by moving a finite distance for any non-zero initial velocity.

We shall give the conditions, under which the network of dynamical agents (1) achieve asymptotical consensus meaning that there exists a fixed position (equilibrium) $x^{*} \in \mathrm{R}^{2}$ such that for $i \in M$

$$
\begin{aligned}
& \lim _{t \rightarrow \infty} x_{i}(t)=x^{*} \\
& \lim _{t \rightarrow \infty} v_{i}(t)=0_{2 \times 1}
\end{aligned}
$$

Due to time-delay in communicated network, the control protocol of the dynamical agent $p_{i}$ is a neighborbased linear control law in the form that

$$
u_{i}=\sum_{p_{j} \in N_{i}} a_{i j}\left(y_{j}\left(t-\tau_{i j}(t)\right)-y_{i}\left(t-\tau_{i j}(t)\right)\right)
$$

where $N_{i}$ is the set of neighbors of agent $p_{i}$ and $a_{i j}$ are adjacency elements of $A$. The $\tau_{i j}(t) \geq 0$, denoting the communication transmission time-delay from agent $p_{j}$ to agent $p_{i}$. In this paper, we discuss the consensus of dynamical agent under the condition of the communicated error and the time delay. We focus on the simplest possible case where the time-delays in all channels are equal to $\tau$ and the communicated errors are equal to $\sigma$.

Remark 1. If we choose $\sigma=0$ and $k_{11}=k_{22}=k$, $k_{12}=k_{21}=0$, then the two-dimension agent systems (1) with the control protocol (3) can be decoupled into two identical linear systems and it was discussed by some literatures with or without time delays( such as $[8,9]$ ).

\section{Consensus of Dynamic Agents}

We denote the initial locations and the initial velocities of the agents by

$$
x(0)=\left(x_{1}^{T}(0) \cdots x_{M}^{T}(0)\right)^{T}, \quad v(0)=\left(v_{1}^{T}(0) \cdots v_{M}^{T}(0)\right)^{T}
$$

respectively. Under control protocol (3) with $\tau_{i j}(t)=\tau$, the dynamical equation of agent $p_{i}$ is written by

$$
\dot{\xi}_{i}(t)=A \xi_{i}(t)+B \sum_{p_{j} \in N_{i}} a_{i j}\left(\xi_{j}(t-\tau)-\xi_{i}(t-\tau)\right)
$$

where $\xi_{i}(t)=\left(x_{i}^{T}(t), v_{i}^{T}(t)\right)^{T}, \quad i \in \underline{M}$

$$
A=\left[\begin{array}{cc}
0_{2 \times 2} & I_{2 \times 2} \\
0_{2 \times 2} & K
\end{array}\right], \quad B=\left[\begin{array}{cc}
0_{2 \times 2} & 0_{2 \times 2} \\
I_{2 \times 2} & 0_{2 \times 2}
\end{array}\right] .
$$

Furthermore, let $\xi(t)=\left(\xi_{1}^{T}(t), \cdots, \xi_{M}^{T}(t)\right)^{T}$, then the dynamic network is of the following form

where

$$
\dot{\xi}(t)=\Upsilon_{1} \xi(t)-\Upsilon_{2} \xi(t-\tau)
$$

$$
\Upsilon_{1}=I_{M} \otimes A, \quad \Upsilon_{2}=L \otimes B
$$


And $L$ is the Laplacian associated with the connected graph $G$. Because (5) is a standard linear time-delay dynamical systems, its stability analysis is equivalent to analyzing the eigenvalues and eigenvectors of matrix $\Upsilon=\Upsilon_{1}-e^{\tau} \Upsilon_{2}$.

Lemma 1. The matrix $\Upsilon=\Upsilon_{1}-e^{\tau} \Upsilon_{2}$ has two eigenvectors associated with zero eigenvalue. Let $\alpha, \beta$ be the left and right eigenvectors (denoted by matrices) of matrix $\Upsilon$ associated with zero eigenvalue, respectively. Then

$$
\alpha^{T}=\frac{1}{\sqrt{M}} 1_{M} \otimes\left[\begin{array}{c}
K^{T} \\
-I_{2 \times 2}
\end{array}\right], \quad \beta=\frac{1}{\sqrt{M}} 1_{M} \otimes\left[\begin{array}{l}
K^{-1} \\
0_{2 \times 2}
\end{array}\right]
$$

and $\alpha \beta=I_{2 \times 2}$, where $1_{M}=(1, \cdots, 1)^{T} \in R^{M}$.

Proof It is well known that (refer to $[2,11]$ ) the graph $G=(V, E, A)$ is connected if and only if its Laplacian satisfies that $\operatorname{rank}(L)=M-1$. Moreover, $1_{M}=(1, \cdots, 1)^{T}$ is an eigenvector of $\mathrm{L}$ associated with zero eigenvalue. Then, there is only one zero eigenvalue of $L$, all the other ones are positive and real. By the definition of (6) and $\Upsilon=\Upsilon_{1}-e^{\tau} \Upsilon_{2}$, one has

$$
\begin{aligned}
& \alpha \cdot \Upsilon=\left(\frac{1}{\sqrt{M}} 1_{M}^{T} \otimes\left[\begin{array}{ll}
K & -I_{2 \times 2}
\end{array}\right]\right) \cdot\left(\Upsilon_{1}-e^{\tau} \Upsilon_{2}\right) \\
&=\left(\frac{1}{\sqrt{M}} 1_{M}^{T} I_{M} \otimes\left[\begin{array}{ll}
K & -I_{2 \times 2}
\end{array}\right]\left[\begin{array}{cc}
0_{2 \times 2} & I_{2 \times 2} \\
0_{2 \times 2} & K
\end{array}\right]\right) \\
&-\left(\frac{1}{\sqrt{M}} 1_{M}^{T} e^{\tau} L \otimes-e^{\tau} L \otimes\left[\begin{array}{ll}
K & -I_{2 \times 2}
\end{array}\right]\left[\begin{array}{ll}
0_{2 \times 2} & 0_{2 \times 2} \\
I_{2 \times 2} & 0_{2 \times 2}
\end{array}\right]\right) \\
&= 0_{4 M \times 2}^{T}
\end{aligned}
$$

Thus, $\alpha^{T}=\frac{1}{\sqrt{M}} 1_{M} \otimes\left[\begin{array}{c}K^{T} \\ -I_{2 \times 2}\end{array}\right]$ represents the two left eigenvectors of $\Upsilon$.

Similarly, it is easy to check $\Upsilon \cdot \beta=0_{4 M \times 2}$, which implies that $\beta=\frac{1}{\sqrt{M}} 1_{M} \otimes\left[\begin{array}{l}K^{-1} \\ 0_{2 \times 2}\end{array}\right]$ represents two lefteigenvectors of $\Upsilon$. And it is holds that $\alpha \cdot \beta=I_{2 \times 2}$.

Theorem 1 If the dynamical feedback matrix $K$ in dynamical agent (1) satisfies Assumption 1, $\sigma_{i}=\sigma$ in the $C$ of (1) and the time delays $\tau_{i j}(t)=\tau$ in (3) satisfies

with

$$
\sigma_{\min }<\sigma<\sigma_{\max }
$$

$$
\sigma_{\min }=\frac{a b c-\sqrt{d}}{2 e^{\tau} \lambda\left(a^{2}+b^{2}\right)}, \quad \sigma_{\max }=\frac{a b c+\sqrt{d}}{2 e^{\tau} \lambda\left(a^{2}+b^{2}\right)}
$$

where $a=k_{21}-k_{12}, b=-k_{11}-k_{22}, c=k_{11} k_{22}-k_{12} k_{21}$, $d=(a b c)^{2}+4 \lambda e^{\tau}\left(a^{2}+b^{2}\right) b^{2} c$ and $\lambda=\lambda_{M}$ denotes the biggest eigenvalue of matrix $L$, then all of the eigen- values of $\Upsilon=\Upsilon_{1}-e^{\tau} \Upsilon_{2}$ defined in (6) are of negative real parts except for only two zero eigenvalues.

Proof As the graph $G$ is connected, one denotes the eigenvalues of $L$ by $0=\lambda_{1}<\lambda_{2} \leq \cdots \leq \lambda_{M}=\lambda$. There exists an orthogonal matrix $W$ such that

$$
W^{T} L W=\operatorname{diag}\left\{\lambda_{1}, \lambda_{2}, \cdots, \lambda_{M}\right\} .
$$

One can verify the following formulae.

$$
\begin{aligned}
& \left(W^{T} \otimes I_{4 \times 4}\right) \Upsilon\left(W \otimes I_{4 \times 4}\right) \\
& =\left(W^{T} \otimes I_{4 \times 4}\right)\left(I_{M} \otimes A-e^{\tau} L \otimes B\right)\left(W \otimes I_{4 \times 4}\right) \\
& =\left[\begin{array}{cccc}
A-\lambda_{1} e^{\tau} B & 0_{4 \times 4} & \cdots & 0_{4 \times 4} \\
0_{4 \times 4} & A-\lambda_{2} e^{\tau} B & \cdots & 0_{4 \times 4} \\
\vdots & \vdots & \ddots & \vdots \\
0_{4 \times 4} & 0_{4 \times 4} & \cdots & A-\lambda_{M} e^{\tau} B
\end{array}\right]
\end{aligned}
$$

Then the dynamical behavior of the network (1) is characterized by the eigenvalues of $A-\lambda_{i} e^{\tau} B, i \in \underline{M}$.

First we discuss the block with $\lambda_{1}=0$. By Assumption 1 , one has

$$
k_{11}+k_{22}<0, \quad k_{12} k_{21}<k_{11} k_{22}
$$

and $\operatorname{rank}\left(A-\lambda_{1} e^{\tau} B\right)=2$, its four eigenvalues satisfy

$$
s_{1}=s_{2}=0, \operatorname{Re}\left(s_{3}\right)<0, \operatorname{Re}\left(s_{4}\right)<0
$$

For $\lambda_{i}>0$, one has $A-\lambda_{i} e^{\tau} B=\left[\begin{array}{cc}0_{2 \times 2} & I_{2} \\ -\lambda_{i} e^{\tau} C & k\end{array}\right]$. As $\operatorname{rank}(C)=2$, then $\operatorname{rank}\left(A-\lambda_{i} e^{\tau} B\right)=4$. Therefore, $\Upsilon=\Upsilon_{1}-e^{\tau} \Upsilon_{2}$ has only two zero eigenvalues. Consider the characteristic polynomial of $A-\lambda_{i} e^{\tau} B$ for $i \in \underline{M}$.

$$
\begin{aligned}
& \pi_{A-\lambda_{i} B}(s)=\operatorname{det}\left(s I-\left(A-\lambda_{i} e^{\tau} B\right)\right) \\
& =\left|\begin{array}{cccc}
s & 0 & -1 & 0 \\
0 & s & 0 & -1 \\
\lambda_{i} e^{\tau} & \delta \lambda_{i} e^{\tau} & s-k_{11} & -k_{12} \\
-\delta \lambda_{i} e^{\tau} & \lambda_{i} e^{\tau} & -k_{21} & s-k_{22}
\end{array}\right| \\
& =s^{4}+a_{1} s^{3}+a_{2} s^{2}+a_{3} s+a_{4}
\end{aligned}
$$

where

$$
\begin{aligned}
& a_{1}=-k_{11}-k_{22}, \quad a_{2}=k_{11} k_{22}-k_{12} k_{21}+2 \lambda_{i} e^{\tau}, \\
& a_{3}=e^{\tau} \lambda_{i}\left[\delta\left(k_{21}-k_{12}\right)-\left(k_{11}+k_{22}\right)\right], \\
& a_{4}=e^{2 \tau} \lambda_{i}^{2}\left(1+\delta^{2}\right)
\end{aligned}
$$

Construct the Routh array of $\pi_{A-\lambda_{i} B}(s)$

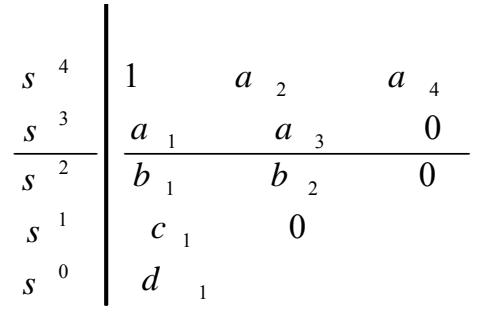


with $b_{1}=\frac{a_{1} a_{2}-a_{3}}{a_{1}}, \quad b_{2}=d_{1}=a_{4}=e^{2 \tau} \lambda_{i}^{2}\left(1-\delta^{2}\right)$,

$$
c_{1}=\frac{b_{1} a_{3}-a_{1} b_{2}}{b_{1}}=\frac{a_{1} a_{2} a_{3}-a_{3}^{2}-a_{1}^{2} a_{4}}{a_{1} b_{1}} .
$$

By the Routh-Hurwith criterion, for stability it is necessary that $a_{1}>0, b_{1}>0, c_{1}>0, d_{1}>0$. Therefore, the dynamical network is stable if and only if the following inequalities hold

$$
\left\{\begin{array}{cc}
a_{1} & >0 \\
a_{4} & >0 \\
a_{1} a_{2}-a_{3} & >0 \\
a_{1} a_{2} a_{3}-a_{3}^{2}-a_{1}^{2} a_{4} & >0
\end{array}\right.
$$

By (10) and (11), it is obvious that the first and second inequalities in (12) hold, and one may easily verify that the third inequality in (12) holds only if the fourth inequalities holds. Then

$$
\begin{aligned}
& a_{1} a_{2} a_{3}-a_{3}^{2}-a_{1}^{2} a_{4} \\
& =-\left\{( k _ { 1 1 } + k _ { 2 2 } ) \cdot ( k _ { 1 1 } k _ { 2 2 } - k _ { 1 2 } k _ { 2 1 } ) \cdot \left[\delta\left(k_{21}-k_{12}\right)\right.\right. \\
& \left.\left.\quad-\left(k_{11}+k_{22}\right)\right]\right\} e^{\tau} \lambda_{i}+e^{2 \tau} \lambda_{i}^{2} \delta^{2}\left[\left(k_{11}+k_{22}\right)^{2}+\left(k_{21}-k_{12}\right)^{2}\right]
\end{aligned}
$$

So the fourth inequality in (12) can be rewritten as the following form.

$$
b^{2} c+a b c \sigma_{i}-e^{\tau} \lambda_{i}\left(a^{2}+b^{2}\right) \sigma_{i}^{2}>0
$$

where $a=k_{21}-k_{12}, b=-k_{11}-k_{22}, c=k_{11} k_{22}-k_{12} k_{21}$. From it, one may obtain

$$
\frac{a b c-\sqrt{d_{i}}}{2 e^{\tau} \lambda_{i}\left(a^{2}+b^{2}\right)}<\sigma_{i}<\frac{a b c+\sqrt{d_{i}}}{2 e^{\tau} \lambda_{i}\left(a^{2}+b^{2}\right)}
$$

where $d_{i}=(a b c)^{2}+4 \lambda_{i} e^{\tau}\left(a^{2}+b^{2}\right) b^{2} c$. Under the case, the matrix $A-\lambda_{i} e^{\tau} B$ is Hurwith. Then it is obvious that the matriies $A-\lambda_{i} e^{\tau} B$ are Hurwith for all $i=2, \cdots, M$ under the condition of (8) and (9). Therefore, all of the eigenvalues of the matrix $\Upsilon=\Upsilon_{1}-e^{\tau} \Upsilon_{2}$ defined in (6) are of negative real parts except for only two zero eigenvalues.

Theorem 2 Under conditions of Theorem 1, the control protocol (3) globally and asymptotically achieves the collective behavior of the dynamic agents.

Proof Under conditions of Theorem 1, all of the eigenvalues of the matrix $\Upsilon=\Upsilon_{1}-e^{\tau} \Upsilon_{2}$ defined in (6) are of negative real parts except for only two zero eigenvalues. By $\Psi:=\left(\theta_{1}, \theta_{2}, \cdots, \theta_{4 M-1}, \theta_{4 M}\right) \in R^{4 M \times 4 M}$ one denotes right-eigenvectors of $\Upsilon$ associated with eigenvalues $\gamma_{1}, \gamma_{2}, \cdots, \gamma_{2 M}$ respectively.

It holds that $\Upsilon \Psi=\Psi \Lambda, \Lambda=\operatorname{diag}\left\{0_{2 \times 2}, J_{1}, \cdots, J_{M}\right\}$, $J_{1}$ denotes the Jordan form of two order associated with the eigenvalues $\gamma_{1}$ and $\gamma_{2} . J_{i}$ denotes the Jordan form of four order associated with the eigenvalues $\gamma_{4 i-3}, \gamma_{4 i-2}, \gamma_{4 i-1}$ and $\gamma_{4 i}$ for all $i=2, \cdots, M$.

Let $\quad \Psi^{-1}=\tilde{\Psi}:=\left(\tilde{\theta}_{1}^{T}, \tilde{\theta}_{2}^{T}, \cdots, \tilde{\theta}_{4 M-1}^{T}, \tilde{\theta}_{4 M}^{T}\right)^{T} \in R^{4 M \times 4 M}$, where $\tilde{\theta}_{i} ; i \in \underline{4 M}$ are $4 M$ row left-eigenvectors of $\Upsilon$ correspondingly. Then it is holds that $\tilde{\Psi} \cdot \Upsilon=\tilde{\Psi} \Lambda$.

As $0>\operatorname{Re}\left(\gamma_{3}\right) \geq \operatorname{Re}\left(\gamma_{4}\right) \geq \cdots \geq \operatorname{Re}\left(\gamma_{4 M-1}\right) \geq \operatorname{Re}\left(\gamma_{4 M}\right)$, one has $\lim _{t \rightarrow \infty} e^{J t}=\left[\begin{array}{cc}I_{2 \times 2} & 0_{2 \times(4 M-2)} \\ 0_{(4 M-2) \times 2} & 0_{(4 M-2) \times(4 M-2)}\end{array}\right]$ and

$$
\lim _{t \rightarrow \infty} \exp (\Upsilon t)=\Psi\left[\begin{array}{cc}
I_{2 \times 2} & 0_{2 \times(4 M-2)} \\
0_{(4 M-2) \times 2} & 0_{(4 M-2) \times(4 M-2)}
\end{array}\right] \tilde{\Psi}
$$

Let $\alpha=\left(\begin{array}{ll}\theta_{1} & \theta_{2}\end{array}\right)$ and $\beta=\left(\begin{array}{c}\tilde{\theta}_{1} \\ \tilde{\theta}_{2}\end{array}\right)$, one has $\lim _{t \rightarrow \infty} \exp (\gamma t)$ $=\beta \alpha$.

Due to the fact that $\Psi \cdot \Psi^{-1}=\Psi \cdot \tilde{\Psi}=I_{4 M \times 4 M}, \quad \alpha$ and $\beta$ satisfy the property $\alpha \beta=I_{2 \times 2}$.

$$
\begin{aligned}
& \lim _{t \rightarrow \infty} \xi(t)=\lim _{t \rightarrow \infty} \exp (\Upsilon t) \xi(0)=\beta \alpha \xi(0) \\
& =\frac{1}{M}\left(\mathbf{1}_{M} \otimes\left[\begin{array}{c}
k^{-1} \\
0_{2 \times 2}
\end{array}\right]\right) \cdot\left(\mathbf{1}_{M}^{\tau} \otimes\left[\begin{array}{ll}
k & -I_{2}
\end{array}\right]\right) \xi(0) \\
& =\frac{1}{M} \mathbf{1}_{M} \mathbf{1}_{M}^{\tau} \otimes\left[\begin{array}{cc}
I_{2} & -k^{-1} \\
0_{2 \times 2} & 0_{2 \times 2}
\end{array}\right] \cdot \xi(0)
\end{aligned}
$$

Therefore, $\quad \lim _{t \rightarrow \infty} x_{i}(t)=\frac{1}{M}\left\{\sum_{j=1}^{M}\left[x_{j}(0)-k^{-1} v_{j}(0)\right]\right\} \quad$ and it is obvious that

$$
\lim _{t \rightarrow \infty} v_{i}(t)=0, \quad i \in\{1,2, \cdots, M\}
$$

This implies the protocol (3) globally asymptotically achieves the collective behavior of the dynamic agents.

Corollary 1 The dynamical feedback matrix $\mathrm{K}$ is chosen to be $k_{12}=k_{21}=k_{1}$ and $k_{11}=k_{22}=k_{2}$, then the control protocol (3) globally and asymptotically achieves the collective behavior of the dynamic agents if the time delays $\tau$ satisfy $\tau<\ln \frac{k_{2}^{2}-k_{1}^{2}}{\lambda \delta^{2}}$.

Proof It is easy to verify according to the proof of Theorem 1 and Theorem 2. Under Assumption 1, one has $k_{12} k_{21}<k_{11} k_{22}$. Thus, one finds that $c>0$ and it further implies that $\sigma_{\min }<0$ and $\sigma_{\max }>0$ in (9) for bounded time delay. Thus we have the following result.

Corollary 2 The dynamical agents achieve collective behavior if the network communicated error $\sigma$ is sufficient small for the bounded time delays.

\section{Simulations}

Numerical simulations will be given to illustrate the theoretical results obtained in the previous section. Con- 
sider five dynamic agents under network described in Figure 1.

We can obtain the Laplacian matrix $L$ of the graph $G$ of Figure 1 and its eigenvalues are $\lambda_{1}=0, \lambda_{2}=\lambda_{3}=$ $\frac{1}{2}(7-\sqrt{13}), \quad \lambda_{4}=\lambda_{5}=\frac{1}{2}(7+\sqrt{13}), \quad \lambda_{6}=4$.

We consider that the dynamic agent (1) in the network has $k=\left[\begin{array}{cc}-1 & 0.1 \\ 0.2 & -1\end{array}\right], \quad \sigma=0.1$ in the observation matrix $C$ and the time delay $\tau=0.1$ in (3). Thus, it is Lyapunov stable and satisfies Assumption 1. One can get $a=0.1$, $b=2, c=0.98, \sqrt{\Delta}=12.1324$ and the $\sigma=0.1$ belongs to the range of parameters, i.e.,

$$
\sigma_{\min }=-0.2125<\sigma<0.2764=\delta_{\max }
$$

When a control protocol (3) is applied into the agents in network, the collective behavior of dynamic agents takes place according to our result. Figures 2-3 give simulation results of the collective behavior of the agents while their velocities.

\section{Conclusion}

In this paper, we discuss the collective behavior of dynamical agents in network which associated with a graph

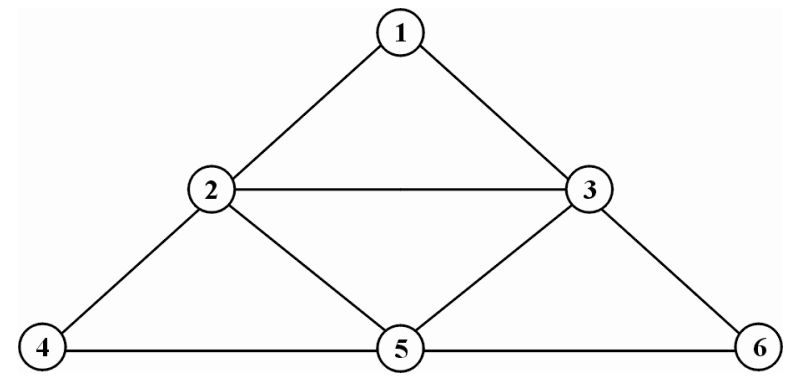

Figure 1. An undirected graph $G$ with $M=6$ nodes.

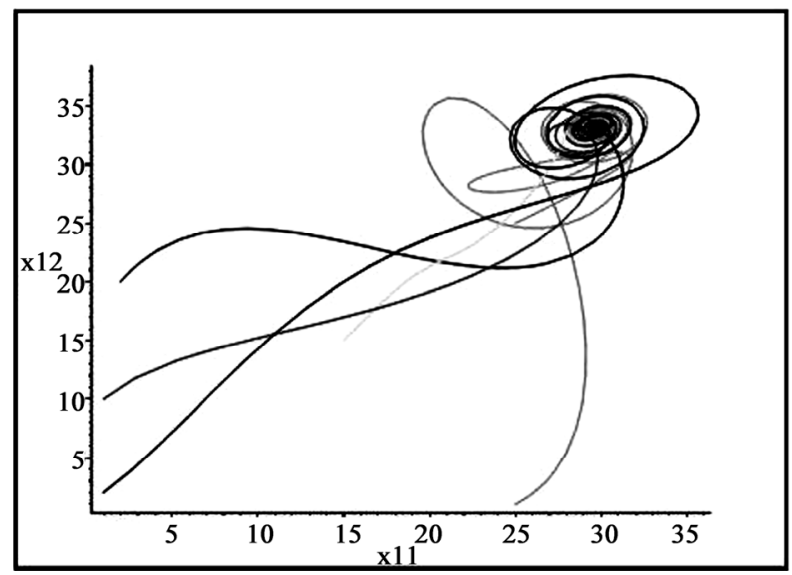

Figure 2. State trajectories of the agents in $G$.

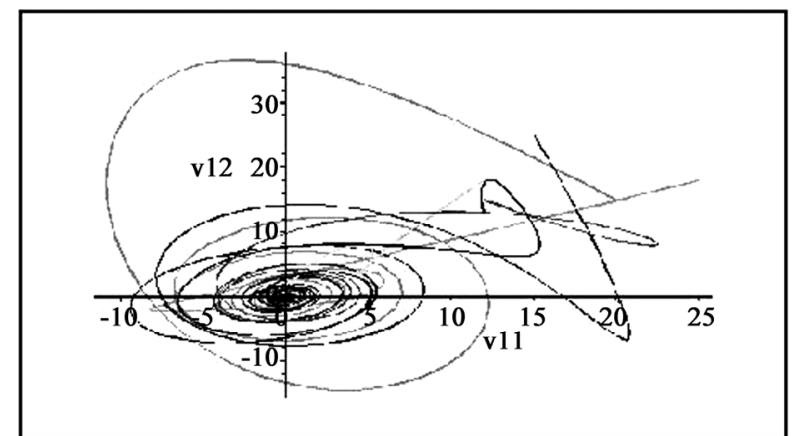

Figure 3. Velocity trajectories of the agents in $G$.

$G$. It is assumed that the agents are Lyapunov stable distributed on a plane and their location coordinates are measured by some remote sensor with certain error and transmitted to its neighbors. Based on the transmitted information with time-delay, the control protocol is designed as a linear decentralized law. The coordination of dynamical agents is shown under the condition that the error is small enough. There is a tradeoff between robustness of a protocol to time-delays and the sensor error.

\section{Acknowledgement}

This work is supported by the Nanjing Audit University Fund (No. 20720035).

\section{References}

[1] A. Jadbabaie, J. Lin and S. A. Morse, "Coordination of Groups of Mobile Autonomous Agents Using Nearest Neighbor Rules," IEEE Transactions on Automatic Control, Vol. 48, No. 6, June 2003, pp. 988-1000.

[2] R. O. Saber and R. M. Murray, "Consensus Problems in Networks of Agents with Switching Topology and TimeDelays," IEEE Transactions on Automatic Control, Vol. 49, No. 9, September 2004, pp. 1520-1533.

[3] R. O. Saber, J. A. Fax and R. M. Murray, "Consensus and Cooperation in Networked Multiagent Systems," Proceedings of the IEEE, Vol. 95, No. 1, January 2007, pp. 215-240.

[4] P. Lin and Y. Jia, "Average Consensus in Networks of Multi-Agents with Both Switching Topology and Coupling Time-Delay," Physica A: Statistical Mechanics and Its Applications, Vol. 387, No. 1, January 2008, pp. 303-313.

[5] Y. Liu and K. M. Passino, "Stable Social Foraging Swarms in a Noisy Environment," IEEE Transactions on Automatic Control, Vol. 49, No. 1, 2004, pp. 30-44.

[6] V. Gazi and K. M. Passion, "Stability Analysis of Social Foraging Swarms," IEEE Transactions on System, Man and Cybernetics, Vol. 34, No. 1, 2004, pp. 539-557.

[7] H. Yu and Y. Zheng, "Global Behavior of Dynamical 
Agents in Directed Network," Proceedings of the 26th Chinese Control Conference, 2007, pp. 557-561.

[8] G. Xie and L. Wang, "Consensus Control for a Class of Networks of Dynamic Agents," International Journal of Robust and Nonlinear Control, Vol. 16, 2006, pp. 1-16.

[9] L. Yang, H. Yu and Y. Zheng, "Aggregation of Dynamical
Agents in Network," 7th World Congress on Intelligent Control and Automation, June 2008, pp. 6348-6352.

[10] N. Biggs and Algebraic, "Graph Theory," Cambridge University Press, Cambridge, 1994.

[11] R. A. Horn and C. R. Johnson, "Matrix Analysis," Cambridge University Press, Cambridge, 1987. 